\title{
A case of Pott's disease with epidural abscess and probable cerebral tuberculoma following Bacillus Calmette-Guérin therapy for superficial bladder cancer
}

\author{
Colin B Josephson MSc MD ${ }^{1}$, Saleh Al-Azri MD², Daniel J Smyth MD $^{3}$, \\ David Haase MBBS FRCPC ${ }^{2}$, B Lynn Johnston MD FRCPC ${ }^{2}$
}

CB Josephson, S Al-Azri, DJ Smyth, D Haase, BL Johnston. A case of Pott's disease with epidural abscess and probable cerebral tuberculoma following Bacillus Calmette-Guérin therapy for superficial bladder cancer. Can J Infect Dis Med Microbiol 2010;21(1):e75-e78.

Intravesical Bacillus Calmette-Guérin (BCG) immunotherapy is an accepted treatment for transitional cell carcinoma of the bladder. Carcinoma in situ of the bladder progresses to invasive muscular disease in approximately $54 \%$ of untreated patients, mandating early initiation of therapy once the diagnosis is confirmed. Should BCG treatment fail, an additional course of BCG combined with interferonalpha, both administered intravesically, is a promising second-line immunotherapy. In greater than $95 \%$ of patients, BCG is tolerated without significant morbidity or mortality. However, both early (within three months of the original treatment) and late presentations of systemic infection resulting from intravesical BCG treatment have been described. The present study describes the course of a 75-year-old man with a late presentation of BCG vertebral osteomyelitis, discitis, epidural abscess, bilateral psoas abscesses and probable cerebral tuberculoma, following treatment regimens of intravesical BCG followed by intravesical BCG plus interferon-alpha $2 \mathrm{~b}$.

\author{
Mal de Pott avec abcès épidural et tuberculome \\ cérébral probable consécutifs à une \\ immunothérapie au bacille Calmette-Guérin \\ pour un cancer superficiel de la vessie
}

\begin{abstract}
L'immunothérapie intravésicale au BCG (bacille Calmette-Guérin) est un traitement accepté pour le carcinome à cellules transitionnelles de la vessie. Le carcinome in situ de la vessie évolue vers une maladie musculaire invasive chez environ $54 \%$ des patients non traités, ce qui justifie l'instauration rapide d'un traitement une fois le diagnostic confirmé. Si le traitement au BCG échoue, un second cycle, allié cette fois à l'interféron alpha, tous deux administrés par voie intravésicale, constitue une immunothérapie de seconde intention prometteuse. Chez plus de $95 \%$ des patients, le BCG est toléré, sans morbidité ni mortalité significatives. Toutefois, des tableaux précoces (dans les trois mois suivant le traitement initial) et tardifs d'infection systémique consécutive à l'immunothérapie intravésicale au BCG ont été décrits. La présente étude montre l'évolution d'un patient de 75 ans porteur d'un tableau tardif d'ostéomyélite vertébrale, discite, abcès épidural, abcès bilatéraux des psoas et tuberculome cérébral probable associés au BCG consécutif à une immunothérapie intravésicale au BCG, répétée ensuite avec l'interféron-alpha $2 \mathrm{~b}$.
\end{abstract}

Key Words: Intravesical BCG; IFN- $\alpha 2 b$; Pott's tuberculoma

Since its initial description in 1976 (1), intravesical Bacillus $\checkmark$ Calmette-Guérin (BCG) immunotherapy has become an accepted treatment for transitional cell carcinoma of the bladder. Studies $(2,3)$ have demonstrated that carcinoma in situ of the bladder progresses to invasive muscular disease in approximately $54 \%$ of untreated patients, mandating early initiation of therapy once the diagnosis is confirmed. The complete response rate to intravesical BCG, a live attenuated strain of Mycobacterium bovis, averages $72 \%$ and, with the addition of three weekly treatments at three-month intervals, $85 \%$ of those without invasive disease are expected to make a complete recovery by six months (3). Should BCG treatment fail, there is phase II evidence that an additional course of BCG combined with interferon-alpha (IFN- $\alpha$ ), both administered intravesically, is a promising secondline immunotherapy $(4,5)$. In greater than $95 \%$ of patients, BCG is tolerated without significant morbidity or mortality (6). However, both early (within three months of the original treatment) and late presentations of systemic infection resulting from intravesical BCG treatment have been described (7). We describe the course of a 75-year-old man with a late presentation of BCG vertebral osteomyelitis, discitis, epidural abscess, bilateral psoas abscesses and probable cerebral tuberculoma, following treatment regimens of intravesical BCG followed by intravesical BCG plus IFN- $\alpha 2 b$.

\section{CASE PRESENTATION}

A 75-year-old man with a history of stage $1 \mathrm{~A}$ mycoses fungoides in remission since 2004, initially came to medical attention in the spring of 2005 with a one-month history of gross hematuria, frequent urination, decreased urinary flow, and a sensation of incomplete voiding and irritation at the glans penis. Kidney and abdominal ultrasound revealed a renal cyst and a mass in the bladder. Cystoscopic examination revealed multiple lesions lining the bladder wall, and biopsy showed papillary urothelial carcinoma with high-grade nuclear features. Histology was consistent with grade 3 transitional cells. There was focal invasion of the lamina propria without involvement of the muscularis propria.

The patient underwent transurethral resection of multiple bladder tumours followed two weeks later by a six-week course

${ }^{1}$ Divisions of Neurology; ${ }^{2}$ Infectious Disease; ${ }^{3}$ Department of Medicine, Dalhousie University, Halifax, Nova Scotia

Correspondence and reprints: Dr B Lynn Johnston, Division of Infectious Disease, Department of Medicine, Dalhousie University, Room 5014,

Dickson Building, Queen Elizabeth II Health Sciences Centre, 1278 Tower Road, Halifax, Nova Scotia B3H 2Y9. Telephone 902-473-5553,

fax902-473-7394, e-mail ljohnsto@dal ca 


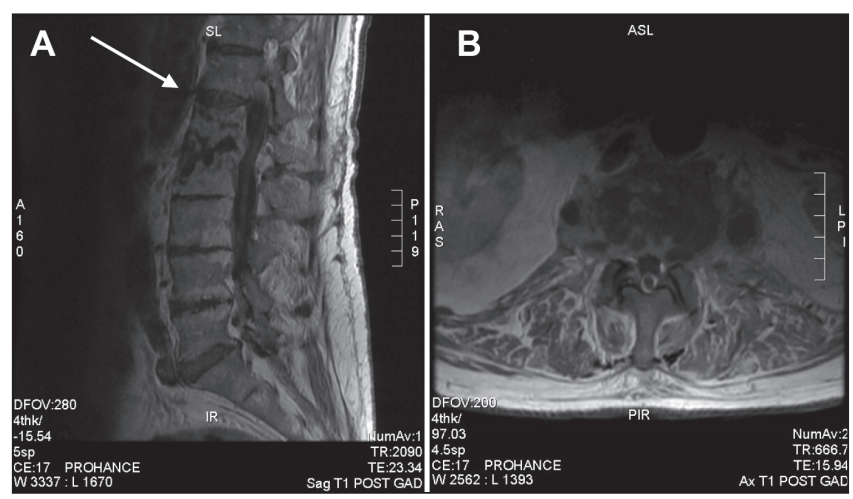

Figure 1) Magnetic resonance imaging. Sagittal T1 fluid attenuation inversion recovery (A) and axial T1 (B) postgadolinium demonstrating L1/L2 discitis (arrow) with extension into the epidural space, mild thecal sack compression and bilateral psoas muscle inflammation

of intravesical BCG-Tice strain (OncoTICE, Organon Laboratories Limited, United Kingdom), $50 \mathrm{~mL}$ per treatment with one treatment per week. After completing the full course of BCG therapy, routine cystoscopy was performed during which two biopsies were taken. The first specimen showed papillary urothelial carcinoma with high-grade nuclear features, but no invasion of the lamina or muscularis propria. The second sample was negative for dysplasia or malignancy.

On the basis of the biopsy results, a decision was made to start combined intravesical BCG and IFN- $\alpha$ therapy. Starting August 3,2005 , the patient received six weeks of once weekly intravesical BCG $(17 \mathrm{~mL})$ and $50 \times 10^{6}$ units of IFN- $\alpha 2 \mathrm{~b}$. Follow-up cystoscopy was negative for dysplasia or malignancy. Maintenance therapy was then initiated with an intended schedule of three weeks of once weekly treatment every six months for the next two years. On two occasions in November 2006, and once in October 2007, difficult catheter insertion was noted, but the treatment proceeded. The patient had cystoscopy performed on several occasions after his intravesical treatments, and no significant abnormalities were noted in the bladder mucosa.

A total of three cycles of maintenance therapy (May and November 2006, and October 2007) had been completed, during which time the patient began to have a slow decline in his level of function. Over a six-month period, he complained of increasing low back pain, generalized weakness, a depressed mood and 'cognitive slowing'. This decline accelerated over the two to three months before his presentation, such that this previously independent man became completely dependent for most daily living activities. Just before admission, he began to experience occasional $30 \mathrm{~min}$ episodes of disorientation and confusion. On the day of admission, he suffered a fall from a seated position, and thus was brought to the emergency department where he was admitted to the general medicine ward.

No abnormalities were noted on physical examination apart from 3/5 strength bilaterally in his hip flexors and extensors, believed to be secondary to pain. Routine cell counts and electrolytes were normal, with the exception of a normocytic anemia (hemoglobin level of $128 \mathrm{~g} / \mathrm{L}$ ). Computed tomography (CT) scan of his head showed enlarged ventricles and a paucity of parasagittal sulcal markings. Plain x-rays of his cervical, thoracic and lumbar (L) spine revealed bony destruction at the L1/L2 vertebral level, suggestive of discitis. A chest $\mathrm{x}$-ray and

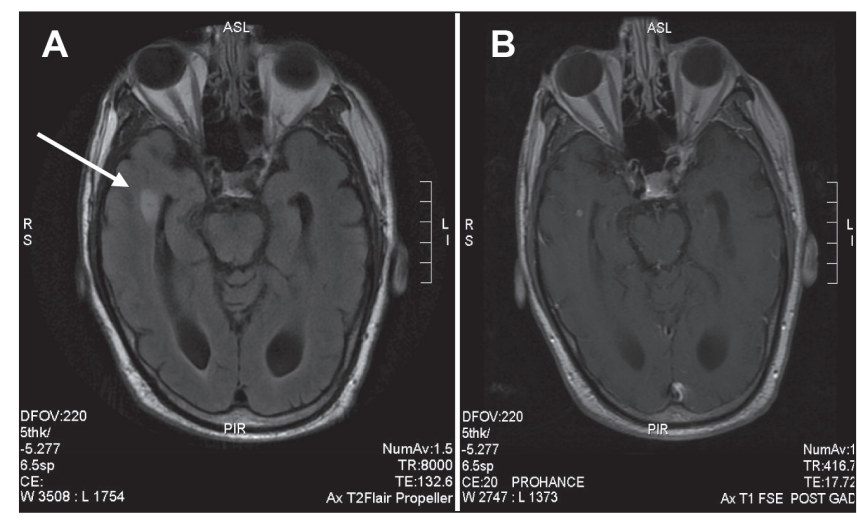

Figure 2) Axial T2 fluid attenuation inversion recovery (A) and axial T1 (B) postgadolinium demonstrating a new right temporal lobe-enhancing lesion presumed to be a tuberculous granuloma (arrow)

CT scan of his chest, abdomen and pelvis were both normal aside from benign cysts in his liver and kidney. A CT scan of his lumbar spine revealed a lytic process centred at the L1/L2 disc space. There was involvement of the adjacent vertebral bodies in association with a soft tissue density closely approximated to the left psoas muscle that contained multiple hypoattenuated lesions, highly suggestive of discitis or osteomyelitis and psoas abscess. Head magnetic resonance imaging (MRI) revealed only cerebral atrophy and large ventricles.

Multiple bacterial blood cultures were negative. MRI of the spine revealed L1/L2 discitis with extension into the epidural space down to the level of L2/L3 (Figure 1). There was a mild degree of thecal sac compression with associated inflammation related to bilateral psoas muscles abscesses. Piperacillin-tazobactam was started at a dose of $3.375 \mathrm{mg}$ every $6 \mathrm{~h}$, and a CT-guided aspiration was arranged. Initial aerobic/anaerobic culture of the aspirate was negative after five days, as was $16 \mathrm{~S}$ ribosomal DNA polymerase chain reaction (PCR). After 11 days of therapy, the piperacillin-tazobactam was changed to intravenous cefazolin $2 \mathrm{~g}$ every $8 \mathrm{~h}$. Fifteen days after the aspirate was obtained, the infectious diseases department was consulted because growth of acidfast microorganisms, subsequently identified as Mycobacterium tuberculosis complex by Gen-Probe DNA probe (Gen-Probe Inc, USA), was noted on mycobacterial culture.

The neurosurgical team believed that there was no indication for invasive management. Pending susceptibility results, isoniazid (300 mg), rifampin (600 mg), pyrazinamide ( $2 \mathrm{~g})$, ethambutol (1.6 g), pyridoxine $(25 \mathrm{mg})$, all once daily, and a tapering dose of dexamethasone (starting at $10 \mathrm{mg}$ four times a day) were started.

When susceptibility results returned from the Canadian Science Centre for Human and Animal Health (Winnipeg, Manitoba) indicating that the organism was resistant to pyrazinamide, that agent was discontinued and the other three were continued. The organism was identified as $M$ bovis BCG using major polymorphic tandem repeat sequencing analysis (8).

The hospital course was prolonged because of immobility and difficulty with pain control. MRI of the head for ongoing episodes of delirium and confusion demonstrated a new enhancing nodule in the right temporal lobe that likely represented a new tuberculous granuloma (Figure 2). Repeat MRI of the spine revealed no change in the epidural abscess, although there was new posterior protrusion of the bony aspect of the L2 vertebral 
body into the spinal canal, resulting in significant spinal stenosis. The neurosurgical team still believed that there was no indication for surgical intervention given the lack of significant findings on physical examination. The patient completed a 12-month course of isoniazid, rifampicin and pyridoxine, after an initial two months of ethambutol, in addition to the other two antituberculous drugs. He has since been discharged to a long-term care facility.

\section{DISCUSSION}

Intravesical BCG is standard therapy for superficial transitional cell carcinoma of the bladder. It is believed to induce an immune response that eradicates bladder cancer through anti-BCG-induced cell-mediated immunity $(9,10)$. Its effects are generally localized to the bladder surface, suggesting that it is the local immune response and the inherent antineoplastic properties of the BCG itself that are primarily responsible for its activity (9). It is considered safe and effective with serious side effects reported in less than $5 \%$ of 2602 patients included in one retrospective analysis (11).

However, as with most antineoplastic agents, there are significant potential toxicities. The most serious of these are systemic infections with the BCG strain. Gonzalez et al (7), in a case series of six patients and review of the literaure, divided post-treatment BCG infection into early and late disease. Early disease (three patients) occurred within eight to 12 weeks of the first BCG treatment and late disease at least one year following the administration of the first BCG dose. Patients with early disease had generalized constitutional symptoms and evidence of systemic spread with hepatitis and pneumonitis in almost every case. Granulomas were present in all biopsied cases, but cultures were positive in fewer than one-half (five of 17) of cases. The interpretation was that early presentation represents systemic infection with a relatively low-grade pathogen in immune-competent hosts. Repeated administration of a low-grade pathogen then results in granuloma formation. Late presentations may result from reactivation after successful control of early dissemination. Granulomas are present in all cases, and cultures are generally positive (10 of 15 cases). It was suggested that disease either occurs at the site of the highest inoculation or at sites typical for extrapulmonary tuberculosis (7).

Vertebral osteomyelitis following intravesical BCG has been previously documented (12-20). Most cases were late presentations in elderly men who had completed their course of BCG. The first case of Pott's disease resulting from intravesical BCG therapy was documented in 1992 (15) and, similar to our case, the vertebral osteomyelitis was also associated with a psoas abscess. We believe that this is the first case with an associated epidural abscess and probable cerebral tuberculoma.

The intravesical administration of IFN- $\alpha$ has proven to be efficacious in the treatment of superficial bladder cancer (3). In vitro studies have demonstrated a pronounced $\mathrm{T}$ helper type 1 immune response within the bladder following BCG administration, with elevations in interleukin 12 and IFN-gamma (21). Higher doses of IFN therapy have been used in patients intolerant of full dose BCG during phase 2 trials. This was not associated with a significant increase in side effects, including BCG dissemination (5).

The limited number of published cases effectively prevents a robust evidence-based evaluation of risk factors for systemic spread of intravesical BCG. Previous reports have suggested concurrent severe cystitis $(11,22)$, traumatic catheterization $(11,23)$, deep bladder tumour resection and high-voiding pressures $(24,25)$, bladder injury during BCG instillation $(23,26)$, pelvic irradiation (23), transurethral tumour resection with or without prostate biopsy (27), and potentially immune compromised states (28). Although rare, acid-fast bacilli may persist in the urinary tract for over one year following treatment (29). This may suggest why some patients, such as the one we have presented, remain at risk even years after treatment. It is possible that the traumatic urethral catheterizations in our patient may have predisposed him to this complication. There is no evidence that he was immune compromised. Stage $1 \mathrm{~A}$ mycoses fungoides is a localized disease and the patient was in remission at the time of the BCG infection, and remains so several months after completing treatment for the BCG infection.

The $M$ tuberculosis complex includes $M$ tuberculosis, $M$ bovis, $\mathrm{M}$ bovis BCG, Mycobacterium africanum, Mycobacterium microti and Mycobacterium canetti. Microscopy and the nucleic acid probe (Gen-Probe) cannot distinguish different members of the complex. However, the patient's lack of contact history and the close proximity of his disease to BCG treatment made the diagnosis of $\mathrm{M}$ bovis BCG most likely. Techniques used to discern $\mathrm{M}$ bovis BCG from its parent strain include high-performance liquid chromatography (30), restriction fragment length polymorphism analysis (31), PCR-based genomic deletion analysis (16), phage typing (32) and amplification of major polymorphic tandem repeat region by PCR, followed by restriction enzyme analysis (33). Unfortunately, these advanced techniques are not available in many clinical laboratories. $M$ bovis and $\mathrm{M}$ bovis $\mathrm{BCG}$ are resistant to pyrazinamide, which is also a clue to the diagnosis. Our patient's isolate was identified as $M$ bovis using partial gyrB gene-specific nucleotide sequencing and confirmed to be $\mathrm{M}$ bovis BCG by analysis of sequenced major polymorphic tandem repeat region (8).

There is no established treatment regimen for disease caused by the BCG strain. It was believed prudent to treat this patient similarly to those with $M$ tuberculosis. With the exception of pyrazinamide, he received standard antituberculous therapy (34). Dexamethasone was included because of its demonstrated benefit in patients with tuberculous meningitis of the brain or spine (35).

Pott's disease, with or without epidural abscess, is a rare complication of intravesical BCG therapy. Although numerous case reports describing BCG dissemination have previously been published, we are not aware of any other cases resulting in both epidural abscess and cerebral tuberculous granuloma. Pott's disease must be considered in the differential diagnosis of those patients who have previously undergone intravesical BCG treatment and subsequently present with new-onset back pain. Further understanding of the risk factors predisposing patients to disseminated BCG following therapy for bladder cancer may help optimize patient selection for this treatment, and inform its optimal use. Instructions for the use of OncoTICE indicate that traumatic catheterization can promote systemic BCG infection and suggest delaying administration for seven to 14 days until mucosal damage has healed (36). Perhaps increased awareness of, and adherence to, this caution may prevent some cases of disseminated BCG infection. 


\section{REFERENCES}

1. Morales A, Eidinger D, Bruce AW. Intracavitary bacillus CalmetteGuérin in the treatment of superficial bladder tumors. J Urol 1976;116:180-3.

2. Kirkali Z, Chan T, Manoharan M, et al. Bladder cancer: Epidemiology, staging and grading, and diagnosis. Urology 2005;66:4-34.

3. Lamm D, Herr H, Jakse G, et al. Updated concepts and treatment of carcinoma in situ. Urol Oncol 1998; 4:130-8.

4. Joudi FN, Smith BJ, O'Donnell MA, National BCG-Interferon Phase 2 Investigator Group. Final results from a national multicenter phase II trial of combination bacillus Calmette-Guérin plus interferon alpha-2B for reducing recurrence of superficial bladder cancer. Urol Oncol 2006;24:344-8.

5. O'Donnell MA, Lilli K, Leopold C, National Bacillus CalmetteGuérin/Interferon Phase 2 Investigator Group. Interim results from a national multicenter phase II trial of combination bacillus Calmette-Guérin plus interferon alfa-2b for superficial bladder cancer. J Urol 2004;172:888-93.

6. Lamm DL. Efficacy and safety of bacille Calmette-Guérin immunotherapy in superficial bladder cancer. Clin Infect Dis 2000;31(Suppl 3):S86-90.

7. Gonzalez OY, Musher DM, Brar I, et al. Spectrum of bacille Calmette-Guérin (BCG) infection after intravesical BCG immunotherapy. Clin Infect Dis 2003;36:140-8.

8. Niemann S, Harmsen D, Rusch-Gerdes S, Richter E. Differentiation of clinical Mycobacterium tuberculosis complex isolates by gyrB DNA sequence polymorphism analysis. J Clin Microbiol 2000;38:3231-4.

9. Prescott S, Jackson AM, Hawkyard SJ, Alexandroff AB, James K. Mechanisms of action of intravesical bacille Calmette-Guérin: Local immune mechanisms. Clin Infect Dis 2000;31(Suppl 3):S91-3.

10. Wittes RC. Immunology of bacille Calmette-Guérin and related topics. Clin Infect Dis 2000;31(Suppl 3):S59-63.

11. Lamm DL, van der Meijden PM, Morales A, et al. Incidence and treatment of complications of bacillus Calmette-Guérin intravesical therapy in superficial bladder cancer. J Urol 1992;147:596-600.

12. Abu-Nader R, Terrell CL. Mycobacterium bovis vertebral osteomyelitis as a complication of intravesical BCG use. Mayo Clin Proc 2002;77:393-7.

13. Aljada IS, Crane JK, Corriere N, Wagle DG, Amsterdam D. Mycobacterium bovis BCG causing vertebral osteomyelitis (Pott's disease) following intravesical BCG therapy. J Clin Microbiol 1999;37:2106-8.

14. Fishman JR, Walton DT, Flynn NM, Benson DR, deVere White RW. Tuberculous spondylitis as a complication of intravesical bacillus Calmette-Guérin therapy. J Urol 1993;149:584-7.

15. Katz DS, Wogalter H, D'Esposito RF, Cunha BA. Mycobacterium bovis vertebral osteomyelitis and psoas abscess after intravesical BCG therapy for bladder carcinoma. Urology 1992;40:63-6.

16. Morgan MB, Iseman MD. Mycobacterium bovis vertebral osteomyelitis as a complication of intravesical administration of bacille Calmette-Guérin. Am J Med 1996;100:372-3.

17. Nikaido T, Ishibashi K, Otani K, et al. Mycobacterium bovis BCG vertebral osteomyelitis after intravesical BCG therapy, diagnosed by PCR-based genomic deletion analysis. J Clin Microbiol 2007;45:4085-7.

18. Rozenblit A, Wasserman E, Marin ML, Veith FJ, Cynamon J, Rozenblit G. Infected aortic aneurysm and vertebral osteomyelitis after intravesical bacillus Calmette-Guérin therapy. AJR Am J Roentgenol 1996;167:711-3.
19. Sugita Y, Chokyu H, Gotoh A, et al. Tuberculous spondylitis after intravesical BCG instillation: A case report. Nippon Hinyokika Gakkai Zasshi 1995;86:1493-6.

20. Mavrogenis AF, Sakellariou VI, Tsiodras S, Papagelopoulos PJ. Late Mycobacterium bovis spondylitis after intravesical BCG therapy. Joint Bone Spine 2009;76:296-300.

21. Luo Y, Chen X, Downs TM, Dewolf WC, O'Donnell MA. IFN- $\alpha$ enhances TH1 cytokine responses in bladder cancer patients receiving Mycobacterium bovis Calmette-Guérin immunotherapy. J Immunol 1999;162:2399-405.

22. Van der Meijden. Practical approaches to the prevention and treatment of adverse reactions to BCG. Eur Urol 1995;27(Suppl 1):23-8

23. Steg A, Leleu C, Debre B, Boccon-Gibod L, Sicard D. Systemic bacillus Calmette-Guerin infection, 'BCGitis', in patients treated by intravesical bascillus Calmette-Guerin therapy for bladder cancer. Eur Urol 1989;16:161-4.

24. Civen R, Berlin G, Panosian C. Vertebral osteomyelitis after intravesical administration of Bacille Calmette-Guerin. Clin Infect Dis 1994;18:1013-4.

25. Hakim S, Heaney JA, Heinz T, Zwolak RW. Psoas abscess following intravesical bacillus Calmette-Guerin for bladder cancer: A case report. J Urol 1993;150:188-9.

26. Palayew M, Briedis D, Libman M, Michel RP, Levy RD. Disseminated infection after intravesical BCG immunotherapy. Detection of organisms in pulmonary tissue. Chest 1993;104:307-9.

27. Lamm DL. Complications of bacillus Calmette-Guerin immunotherapy. Urol Clin North Am 1992;19:565-72.

28. Yossepowitch O, Eggener SE, Bochner BH, Donat SM, Herr HW, Dalbagni G. Safety and efficacy of intravesical bacillus CalmetteGuerin instillations in steroid treated and immunocompromised patients. J Urol 2006;176:482-5.

29. Bowyer L, Hall RR, Reading J, Marsh MM. The persistence of bacilli Calmette-Guerin in the bladder after intravesical treatment for bladder cancer. Br J Urol 1995;752:188-92.

30. Floyd MM, Silcox VA, Jones WD Jr, Butler WR, Kilburn JO. Separation of Mycobacterium bovis BCG from Mycobacterium tuberculosis and Mycobacterium bovis by using high-performance liquid chromatography of mycolic acids. J Clin Microbiol 1992;30:1327-30.

31. van Soolingen D, Hermans PW, de Haas PE, van Embden JD. Insertion element IS1081-associated restriction fragment length polymorphisms in Mycobacterium tuberculosis complex species: A reliable tool for recognizing Mycobacterium bovis BCG. J Clin Microbiol 1992;30:1772-7.

32. Jones WD Jr. Differentiation of known strains of BCG from isolates of Mycobacterium bovis and Mycobacterium tuberculosis by using mycobacteriophage 33D. J Clin Microbiol 1975;1:391-2.

33. Hermans PW, van Soolingen D, van Embden JD. Characterization of a major polymorphic tandem repeat in Mycobacterium tuberculosis and its potential use in the epidemiology of Mycobacterium kansasii and Mycobacterium gordonae. J Bacteriol 1992;147:4157-65.

34. World Health Organization. Treatment of tuberculosis: Guidelines for national programmes. In: WHO Report 2003. Geneva: World Health Organization, 2003.

35. Prasad K, Singh MB. Corticosteroids for managing tuberculous meningitis. Cochrane Database Syst Rev 2008;(1):CD002244.

36. Repchinsky C, ed. Compendium of Pharmaceuticals and Specialties. The Canadian Drug Reference for Health Professionals. Canadian Pharmacists Association, 2009:1629-30. 


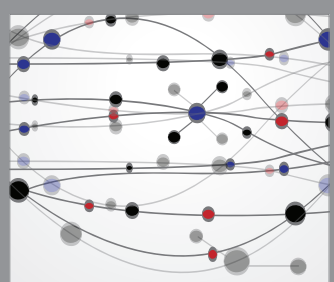

The Scientific World Journal
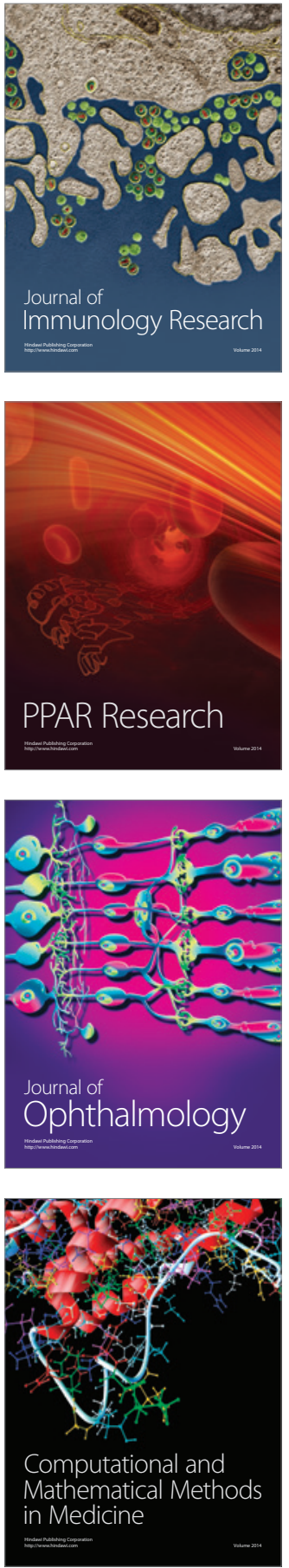

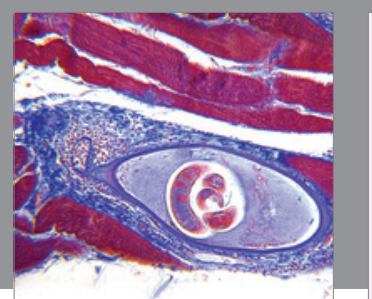

Gastroenterology Research and Practice

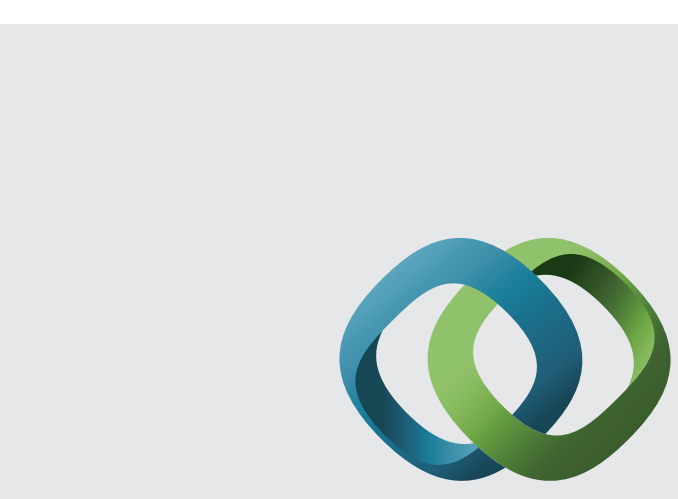

\section{Hindawi}

Submit your manuscripts at

http://www.hindawi.com
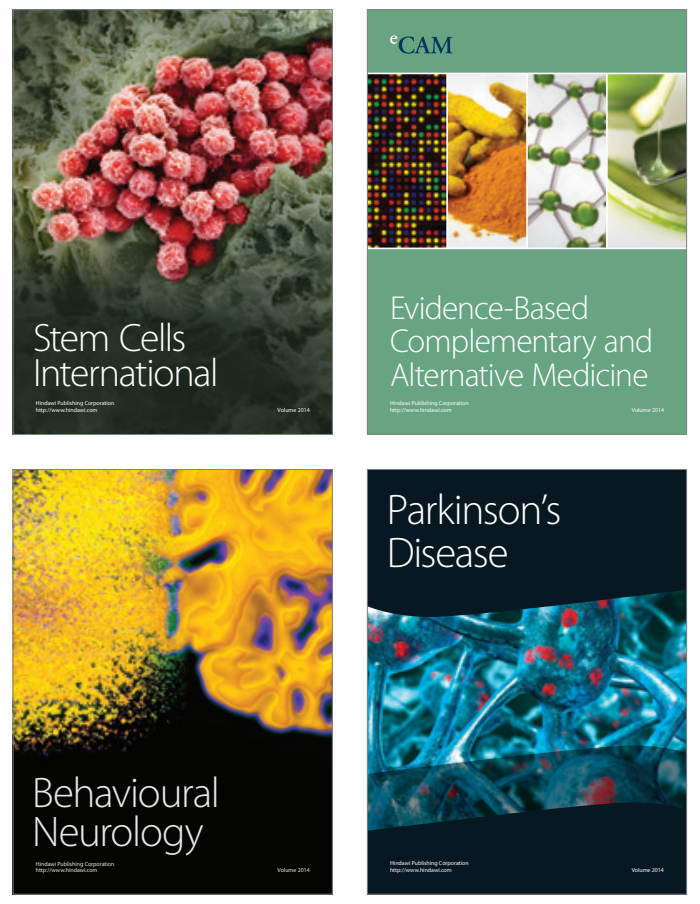
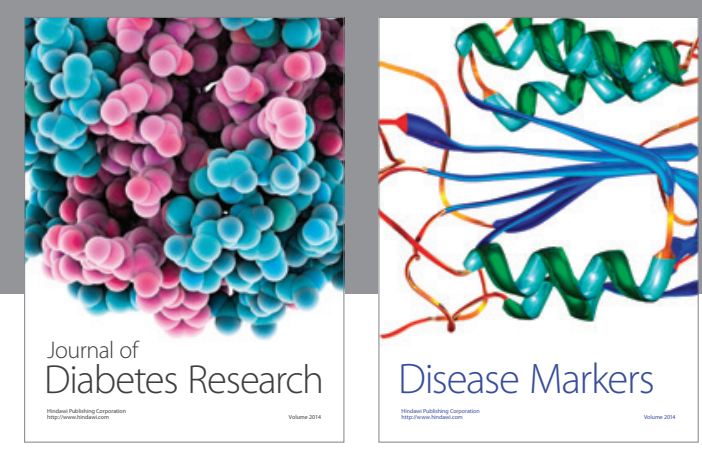

Disease Markers
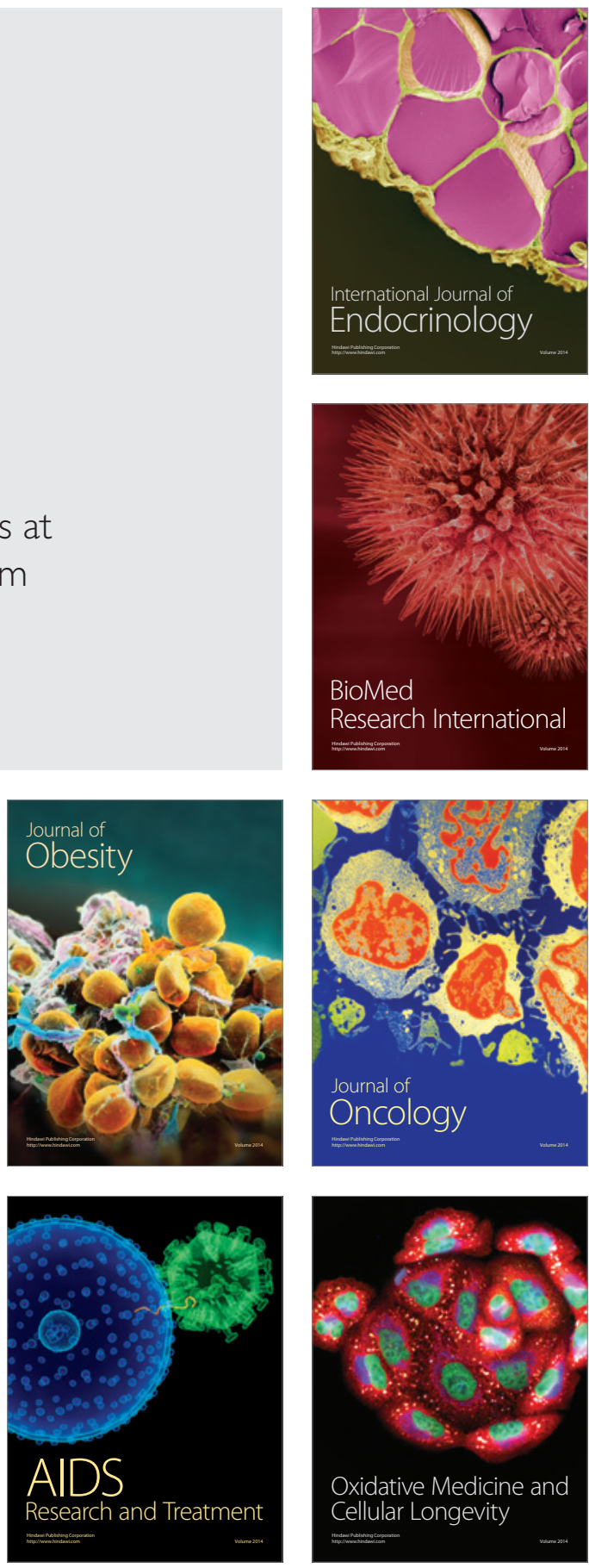